\title{
Effects of Morphology and Interfacial Strength on Mechanical Properties of Ternary Polypropylene Blends with Ethylene-Propylene-Rubber (EPR) and Talc: Molecular Dynamics Study*
}

\author{
Hiroyuki MAE $^{* *}$, Masaki OMIYA ${ }^{* * *}$ and Kikuo KISHIMOTO ${ }^{* * * *}$ \\ "Honda R\&D Co., Ltd. \\ 4630 Shimotakanezawa, Haga-machi, Haga-gun, \\ Tochigi 321-1233, Japan \\ Email: Hiroyuki_Mae@n.t.rd.honda.co.jp \\ *** Department of Mechanical Engineering, Keio University \\ Department of Mechanical and Sciences Engineering, Tokyo Institute of Technology
}

\begin{abstract}
In order to clarify the influences of the morphology and interfacial strength on the microstructural deformation and micro-damage evolution process, a coarse-grained molecular dynamics (MD) simulation is conducted on a mesoscopic specimen of the thermoplastic polypropylene (PP) blended with the ethylene-propylene rubber (EPR) and talc under uniaxial tension. The studied morphologies are two types. The first morphology is composed of three independent phases of PP, EPR and talc. The second morphology consists of core-shell structure of EPR and talc in PP matrix. The first morphology shows that the micro-damage initiates at the interface between PP matrix and talc particle. Then, the micro void is generated there leading to the fibrils of the PP matrix and EPR particles. The second morphology indicates that the micro-damage initiates at the interface between EPR and talc. Meanwhile, the effects of the interfacial strengths of PP-EPR and PP-talc in the first morphology, and PP-EPR and EPR-talc in the second morphology on the macroscopic mechanical properties are further investigated. It appears that the elastic modulus is larger in the first morphology than that in the core-shell structure. It is also found that the maximum stress depends more strongly on the interfacial strength of PP-EPR than the morphological types. It is indicated that the absorbed strain energy is sensitive to both the morphology and the interfacial strength of PP-EPR.
\end{abstract}

Key words: Polymer, Blends, Tensile Behavior, Morphology, Interfacial Strength, Polypropylene, Ethylene Propylene Rubber (EPR), Talc, Molecular Dynamics (MD)

\section{Introduction}

Polypropylene (PP) has been extensively used for automobile applications such as interior and exterior automobile components ${ }^{(1,2)}$ in addition to the field of aerospaces and electric devices because of light weight, ease of molding and resistance to corrosion. Blending PP with inorganic fillers, such as silica ${ }^{(3-5)}$, calcium carbonate ${ }^{(6,7)}$, talc $^{(8-11)}$, clay $^{(12)}$ and wollastonite ${ }^{(13,14)}$, is a useful way to improve physical and mechanical properties of polypropylene composites. The effects of inorganic fillers on the mechanical and physical properties of the PP composites strongly depend on the filler size, shape, aspect ratio, 
interfacial adhesion, surface characteristics and degree of dispersion ${ }^{(5,7,15,16)}$. Talc is one of the most commonly used in-organic fillers in the PP matrix for improving the stiffness of PP blends. Talc fillers induce a significant increase in the starting crystallization temperature of PP and affect the crystallization behavior of talc-filled PP composites ${ }^{(8,17,18)}$. The influences of the crystallinity, molecular weight and lamella thickness on the mechanical properties of PP are studied numerically in the coarse molecular dynamics (MD) simulation by developing the lamella structural model by the same authors ${ }^{(19)}$. The introduction of inorganic fillers to PP leads to an increase in stiffness but a decrease in toughness and ductility $^{(10)}$. The detail analyses of the damage evolution in the talc-filled PP has been studied by Hadal and Misra ${ }^{(13,14)}$. The damage evolution in talc-filled PP is characterized by wedge, ridge-tearing, fibrillation, and brittle fracture with debonding of talc-fillers from the PP matrix, while the damage process of the neat PP is characterized by craze-tearing followed by brittle fracture. From their observations, it is considered that the lack of craze formation in the talc-filled PP is one reason for the loss of toughness and ductility of the neat PP.

It is well known that most semicrystalline polymers can be effectively toughened by incorporation of well-dispersed secondary rubber phase(s) in the matrix. Stamhuis ${ }^{(20)}$ has shown that talc filler can significantly increase the impact resistance of PP if it is physically blended with either an Styrene-Butadiene-Styrene (SBS) or an Ethyrene-Propylene-Diene-Monomer (EPDM) elastomer. The toughening mechanism of rubber-blended PP is the cavitation of rubber particles followed by crazing and shear-band formation in the PP matrix. Rubber particles play a role as an initiator of the craze formation. In addition, the distance between particles improves the toughening of Styrene-Ethylene-Butadiene-Styrene (SEBS) blended PP composites ${ }^{(21-23)}$. However, the significant drawback of rubber toughening is loss of stiffness and strength. Therefore, blending inorganic fillers in the elastomer-blended PP is one solution to achieve the simultaneous improvement of both the stiffness and toughness of the polymer matrix.

The compatibility between the surfaces of the inorganic fillers and PP is also the important factors to improve the physical and mechanical properties ${ }^{(24,25)}$. Velasco et al. ${ }^{(26)}$ shows PP/talc composite with silane coupling agents had a pronounced effect on the crystallization behavior of the PP/talc composites. Gonzalez et al. ${ }^{(6)}$ improved Young's modulus and elongation at break of PP/ High Density Polyethylene (HDPE) blends by using $\mathrm{CaCO}_{3}$ particles treated by different coupling agents. These results indicate that the interfacial strength between inorganic fillers and matrix is the key parameter to enhance the mechanical properties.

Moreover, the phase morphology in the particular relative dispersion of additive components has some effect on the mechanical properties in those PP blends. Two types of the morphologies might be found, either elastomer and filler particles dispersed separately in the PP matrix or elastomer encapsulated filler particles forming core shell inclusion structure. It is expected that these distinct morphologies give the different mechanical properties. The morphologies with a separation structure were reported to have larger stiffness and yield stress than those of the encapsulated filler while the encapsulated morphology exhibited the higher impact absorbed energy ${ }^{(28-30)}$. On the contrary, the contrasting results were obtained by Kolarik et al. ${ }^{(31)}$ and Premphet et al. ${ }^{(32)}$. Kolarik et al. reported that the encapsulated morphology had lower impact absorbed energy than that of a separation structure ${ }^{(31)}$. Premphet et al. showed that the separated morphology had lower yield stress but higher stiffness and impact absorbed energy than the core shell morphology, which was caused by the extensive plastic deformation of PP matrix and cavitation resulted from filler-matrix debonding ${ }^{(32)}$. Thus, it is interesting to investigate the effect of the morphological difference on the mechanical properties in the PP blended with elastomer and inorganic filler. Then, the same authors constructed the coarse MD model where EPR 
and talc were distributed separately in PP matrix while the in situ observation of the microstructural deformation under tensile loading was conducted by transmission electron microscopy $(\mathrm{TEM})^{(33)}$. In the previous study ${ }^{(33)}$, the experimental and numerical simulation results indicated that increasing the interfacial strength between PP and EPR phases was more effective for improving the stiffness and the maximum stress, compared to the PP-talc interfacial strength in the separated morphology. In addition, to enhance the material ductility, it was important to increase the interfacial strength of PP-EPR than that of PP-talc in the case of the separated morphology. However, the effect of the morphological difference has not been analyzed numerically because the previous research focused on only one type of morphology where EPR and talc were separately dispersed in the PP matrix.

Then, the attempt has been made to clarify the influences of the morphology and the interfacial strength on the microstructural deformation and micro-damage evolution process in two types of morphologies (separated and core-shell structures). The base blend system was PP/EPR/talc separated blend because the corresponding morphological MD model was developed in the previous study ${ }^{(33)}$. The tensile deformations were applied in both morphologies and then their mechanical properties were compared. In addition to investigate the morphological effect on the stiffness and toughness, the interfacial strengths of PP-EPR and PP-talc in the separated morphology and those of PP-EPR and EPR-talc in the core-shell morphology were changed parametrically. The effects of those interfacial strengths on the microstructural deformation, the damage evolution process, and the overall mechanical properties were compared. The obtained results are valuable and contributed to design the morphology and the interfacial coupling agents in order to improve both the stiffness and toughness of the polypropylene ternary blends with elastomers and inorganic fillers.

\section{Numerical Procedures}

\subsection{Material}

Isotactic polypropylene (i-PP) was used as a matrix polymer in this study. It has a melt flow rate $(\mathrm{MFR})=30 \mathrm{~g} / 10$ minute $\left(230^{\circ} \mathrm{C}\right)$. The blended elastomer was ethylene propylene rubber (EPR), whose blend ratio was $35 \mathrm{wt} \%$. The talc was blended at the blend ratio of 8 wt \%. Detail of the blend is shown in Table 1. Table 2 shows the detail of EPR. The morphology of the separated PP/EPR/talc blend was obtained by the transmission electron microscopy (TEM) in the previous study ${ }^{(33)}$, as shown in Fig. 1. The EPR particles were elliptical shape to the same direction as the injection direction. The mean diameter of the EPR particles was about $0.7 \mu \mathrm{m}$ based on the image analysis. Based on the TEM morphological picture, the MD model of the base PP/EPR/talc blend was developed ${ }^{(33)}$. Note, that the core-shell type of PP/EPR/talc blend was not experimentally prepared in the present study, inferring that the core-shell morphology was only constructed numerically by modifying the parameters for the phase separation simulation as described in the following section.

Table 1 Detail of constitutes

\begin{tabular}{ccccc}
\hline & Weight ratio & Volume ratio & Density $\left(\mathrm{kg} / \mathrm{m}^{3}\right)$ & Molecular weight $(\mathrm{g} / \mathrm{mol})$ \\
\hline \hline PP & $57 \%$ & $58.9 \%$ & 910 & 190000 \\
EPR & $35 \%$ & $38.3 \%$ & 860 & 320000 \\
Talc & $8 \%$ & $2.8 \%$ & 2700 & - \\
\hline
\end{tabular}

Table 2 Detail of EPR

\begin{tabular}{cccc}
\hline \multicolumn{2}{c}{ Monomer } & Weight ratio & Number of monomers \\
\hline \hline Ethylene & $-\mathrm{C}_{2} \mathrm{H}_{4^{-}}$ & $84.0 \%$ & 9567 \\
Propylene & $-\mathrm{C}_{3} \mathrm{H}_{6^{-}}$ & $16.0 \%$ & 1219 \\
\hline
\end{tabular}




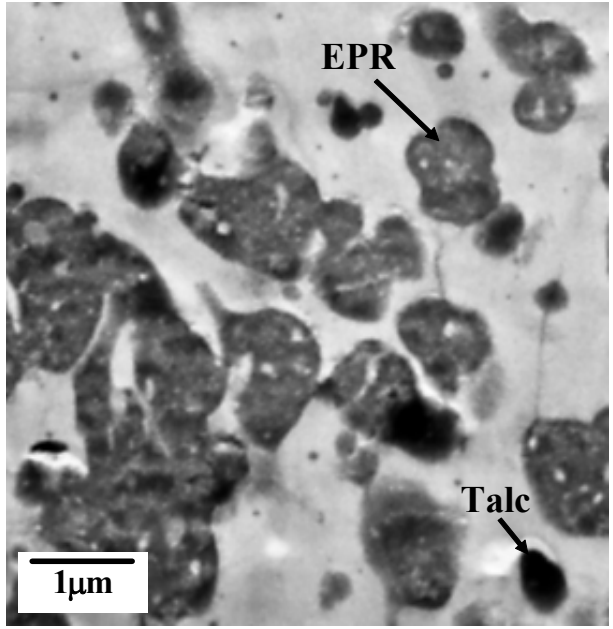

Figure 1 Morphological pictures of separated PP/EPR/talc blend ${ }^{(33)}$

\subsection{Microstructural Phase Separation Simulation}

The material focused in this study was polypropylene blended with EPR and talc. Before simulating the tensile deformation process in such a blend system, one has to conduct the phase separation simulation for constructing the initial simulation models of tensile deformation in the coarse-grained MD.

In this study, the interface simulator SUSHI ${ }^{(34)}$ inside an integrated simulation system for soft materials $\mathrm{OCTA}^{(35)}$ was used. SUSHI calculates the equilibrium and non-equilibrium structures in polymer blends by solving the self-consistent Edwards equation. The static simulations were carried out by SUSHI based on Self Consistent Field Theory $(\mathrm{SCF})^{(36)}$. In the SCF theory, the minimum unit, that constitutes the polymer system, is called a segment. The polymers are composed of several kinds of segments specified by the index $K$. The volume fraction distribution $\phi_{K}(\mathbf{r})$ of $K$-type segments is calculated by taking the chain confirmations into account using the path integral formalism. The self consistent field, $V_{K}(\mathbf{r})$ is a potential field that accounts for the external conditions such as the interaction between segments and the incompressible conditions, and has the following form in this study

$$
V_{K}(\mathbf{r})=W_{K}(\mathbf{r})-\mu_{K}(\mathbf{r}),
$$

where $W_{K}(\mathbf{r})$ and $\mu_{K}(\mathbf{r})$ are the mean field resulting from the interaction between segments and the chemical potential respectively, and are given by

$$
\begin{aligned}
& W_{K}(\mathbf{r})=\sum_{K^{\prime}} \chi_{K K^{\prime}} \phi_{K^{\prime}}(\mathbf{r}), \\
& \mu_{K}(\mathbf{r}) \equiv \delta \Gamma / \delta \phi_{K}(\mathbf{r})
\end{aligned}
$$

acting on a $K$-type segment at position $\boldsymbol{r}$. Here, $\chi_{K K^{\prime}}$ is the interaction strength between the $K$-type segment and $K^{\prime}$-type segment and $\Gamma$ is the free energy in the system. For calculating the full equilibrium state of the distribution of the segment volume fraction, the only constraint condition is the following local incompressible condition.

$$
\sum_{K} \phi_{K}(\mathbf{r})=1 .
$$

In this study, a homo-polymer chain was considered. Then, all the $\mu_{K}(\mathbf{r})$ given by Eq.(3) should be equal irrespective of the segment type $K$.

$$
\mu_{K}(\mathbf{r})=\mu(\mathbf{r}) \text { for all } K
$$

Let us define a quantity $Q_{K}\left(i, \mathbf{r}_{i} ; j, \mathbf{r}_{j}\right)$, so called path integral, as the equilibrium statistical weight of the chain to have a confirmation with the $i$-th and the $j$-th segments at $\mathbf{r}_{\mathrm{i}}$ 
and $\mathbf{r}_{\mathrm{j}}$ positions. Let introduce the path integrals, $Q_{K}\left(i, \mathbf{r}_{i}\right)$ and $\widetilde{Q}_{K}\left(i, \mathbf{r}_{i}\right)$ as follows.

$$
\begin{gathered}
Q_{K}\left(i, \mathbf{r}_{i}\right)=\int d \mathbf{r}_{0} Q_{K}\left(0, \mathbf{r}_{0} ; i, \mathbf{r}_{i}\right) \\
\widetilde{Q}_{K}\left(N_{K}-i, \mathbf{r}_{i}\right)=\int d \mathbf{r}_{N_{K}} Q_{K}\left(N_{K}, \mathbf{r}_{N_{K}} ; i, \mathbf{r}_{i}\right),
\end{gathered}
$$

where $N_{\mathrm{k}}$ is the total number of segments compositing the $K$-type polymer.

Then, the path integrals $Q_{K}\left(i, \mathbf{r}_{i}\right)$ and $\widetilde{Q}_{K}\left(i, \mathbf{r}_{i}\right)$ are obtained by solving the evolution equations

$$
\begin{aligned}
& \frac{\partial}{\partial i} Q_{K}(i, \mathbf{r})=\left[\frac{b^{2}}{6} \nabla^{2}-\beta V_{K}(\mathbf{r})\right] Q_{K}(i, \mathbf{r}) \\
& \frac{\partial}{\partial i} \widetilde{Q}_{K}(i, \mathbf{r})=\left[\frac{b^{2}}{6} \nabla^{2}-\beta V_{K}(\mathbf{r})\right] \widetilde{Q}_{K}(i, \mathbf{r})
\end{aligned}
$$

using the following the initial conditions.

$$
Q_{K}\left(0, \mathbf{r}_{0}\right)=\widetilde{Q}_{K}\left(0, \mathbf{r}_{0}\right)=1
$$

By using the path integrals obtained from Eqs.(8) and (9), the segment density at the position $\mathbf{r}$ is given as follows.

$$
\phi_{K}(\mathbf{r})=C_{K} \sum_{i} Q_{K}(i, \mathbf{r}) \widetilde{Q}_{K}\left(N_{K}-i, \mathbf{r}\right)
$$

where $C_{\mathrm{k}}$ is the normalization constant. In this study, the statistical ensemble of the chains is the canonical ensemble where the total number of chains in the system is fixed and is given as follows.

$$
C_{K}=\frac{M_{K}}{\int d \mathbf{r}_{0} \int d \mathbf{r}_{N_{K}} Q_{K}\left(0, \mathbf{r}_{0} ; N_{K}, \mathbf{r}_{N_{K}}\right)}
$$

Static calculation is conducted for finding a set of self consistent solutions for $\left\{V_{\mathrm{k}}(\mathrm{r})\right\}$ and $\left\{\phi_{K}(\mathbf{r})\right\}$ that satisfy Eqs.(1), (8), (9) and (11), the local incompressible condition Eq.(4), and the constraint force given by Eq.(5) simultaneously. The basic scheme is shown in Fig. 2. These solutions are numerically obtained in SUSHI. The more detail of SUSHI can be found in Ref. (34).

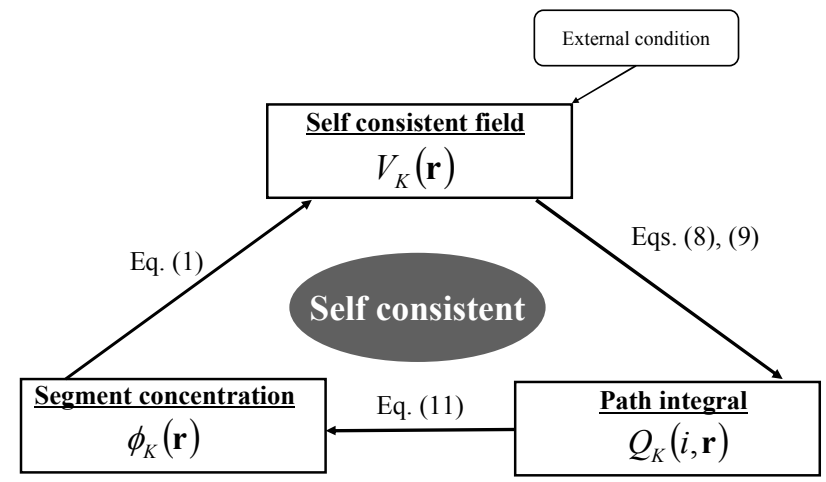

Figure 2 The basic scheme of the SCF theory

The main objective of the present phase separation is to obtain the spatial density distribution of the two types of polymers, which looks like the sea-island morphology. 
Then, the spatial density distributions were obtained by simulating the phase separation in the PP and polyethylene (PE) diblock copolymer blend system. Here, the diblock copolymer means the polymer system consisting of two blocks of polymers. The present PP/PE blend consisted of 13 PP segments and 7 PE segments as illustrated in Fig. 3. Here, talc was modeled as one segment. The phase diagram of diblock copolymer is reported by Matsen, et al. ${ }^{(36)}$, where four types of well known equilibrium mesophases such as spheres, cylinders, gyroid and lamellae are shown. In the phase diagram, the dominant parameters for determining the phase type are the volume fraction $f$ of a segment, the interaction strength $\chi$ between the segments, and the number of segments $N$. In the present study, the target equilibrium phase type was the hexagonal cylindrical shape between PP and PE segments. Then, the interaction strengths were set as shown in Table 3 focusing on the condition $\chi N=20$. Two sets of the interaction parameters between PP segment and talc segment and that between PE segment and talc segment were determined by try and error approaches so that one set should lead to the separated morphology and the other should make the core-shell type of morphology. The unit length $L$ was determined based on the lattice constants of PP/PE diblock copolymer, leading that the lattice constants were $8.0 \mathrm{~L}$ in the $\mathrm{x}$ direction and $14.0 \mathrm{~L}$ in the $\mathrm{y}$ direction in two-dimensional $\mathrm{x}-\mathrm{y}$ plane. The diameter of PE cylindrical domain was about $5 L$. In this study, EPR particles had approximately the diameter of $700 \mathrm{~nm}$. Thus, the unit length $L$ was defined as $140 \mathrm{~nm}$. The system size was $56 L \times 32 L$ as shown in Figs. 4(i-a) and 4(ii-a). The geometry of talc was $3 L \times 7.5 L$. As shown in Figs. 4(i-a) and 4(ii-a), the distribution of the volume fraction of each segment was intentionally applied in the initial phase separation model. The total number of calculation steps was $1,000,000$. During calculation, when the maximum updated amount of $\phi_{K}(\mathbf{r})$ during a single iteration step becomes less than $10^{-6}$, then the iteration procedure was finished. Figures 4(i-b) and 4(ii-b) show the results of phase separated morphology. As shown clearly, the hexagonal cylindrical phase of PE segment in PP matrix was obtained in both morphologies. In addition, the two talc segments were separated from PE segments without constructing the core-shell structures in the morphology A. On the contrary, the morphology B leaded to the core-shell structures of talc and PE in the PP matrix. The obtained distributions of the volume fraction of each segment were used so as to construct the initial structural models for the coarse-grained molecular dynamics as described in next section.

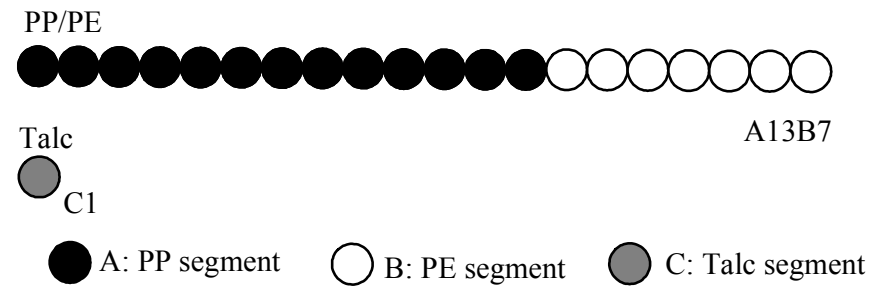

Figure 3 PP/PE diblock copolymer model and talc model

Table 3 Parameters of segment interactions

\begin{tabular}{ccc}
\hline & Morphology A & Morphology B \\
\hline \hline$\chi_{\mathrm{PP} / \mathrm{PE}}$ & 1.0 & 1.0 \\
$\chi_{\mathrm{PP} / \text { Talc }}$ & 1.0 & 1.0 \\
$\chi_{\mathrm{PE} / \text { Talc }}$ & 2.0 & 1.0 \\
\hline
\end{tabular}




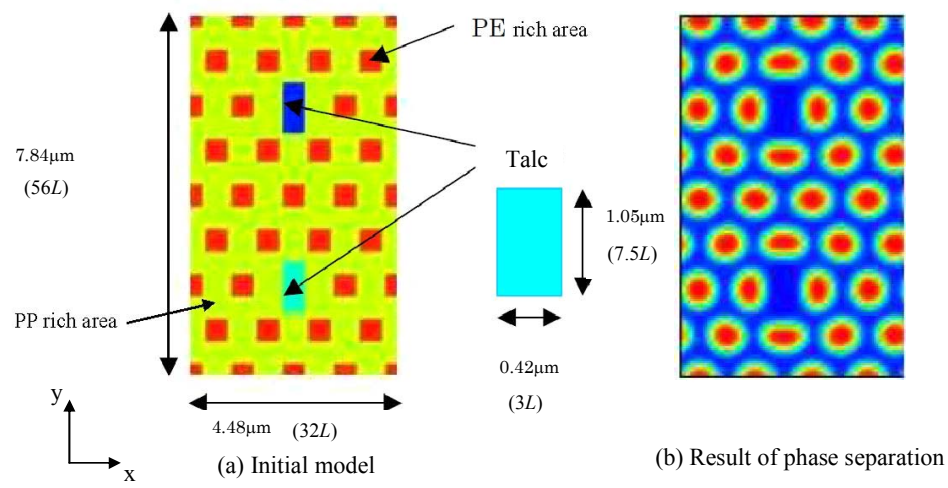

(i) Morphology A

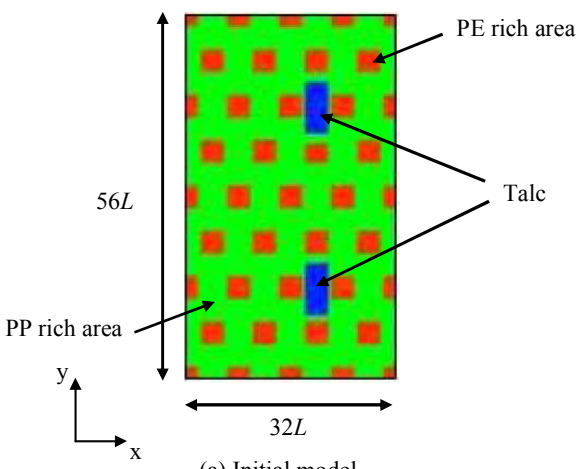

(a) Initial model

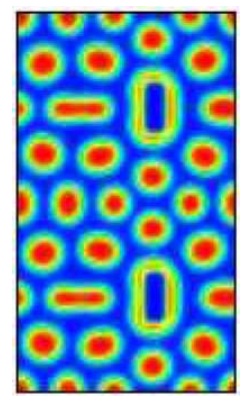

(b) Result of phase separation

(ii) Morphology B

Figure 4 Results of phase separation simulaiton

\subsection{Simulation Method of Tensile Deformation by Coarse-Grained Molecular Dynamics}

In this study, the prototypical system was a dense glass of bead-spring polymer chains filled with soft bead-spring rubbers and stiff talc-like bead-spring inclusions. The system contains three types of particles; PP monomers, PE monomers and inclusion monomers; they were treated as point masses. Reduced units were used throughout while all reduced units were converted for SI units and shown in one table for references. $L$ is the base unit for length, $\rho$ is the reduced density, $P$ is the reduced pressure, $M=\rho L^{3}$ is the base unit for mass, $\tau=L \sqrt{A_{v} M / u_{o}}$ is the base unit for time where $A_{v}$ is Avogadro's number, $u_{0}=P A_{v} L^{3}$ is the base unit for energy and $T=u_{0} / R$ is the reduced temperature where $R$ is the gas constant. The reduced stress will be later mapped to engineering units for parametric study of the interfacial strengths of PP-EPR, PP-talc and EPR-talc. Because of the coarse-grained nature, there is no one-to-one mapping of the model system to the actual system. In this coarse-grained simulation, the goal is to understand the model system self-consistently. In relating the simulation results to the current two types of PP/EPR/talc blends, the emphasis is on the qualitative microstructural deformation and damage evolution mechanisms.

The dynamics was performed using a Langevin thermostat. MD numerically solves Newton's equations of motion for each particle; particles interact according to a potential energy function $U$. We employed a set of potentials to model the polymer chains which are standard for dense bead-spring glasses.

Beads separated by a distance $r$ interact via a truncated Lennard-Jones potential $U_{\mathrm{LJ}}$.

$$
\begin{gathered}
U_{L J}(r)=4 \varepsilon\left[\left(\frac{\sigma_{0}}{r}\right)^{12}-\left(\frac{\sigma_{0}}{r}\right)^{6}\right], r \leq r_{c} \\
U_{L J}(r)=0 \quad, \quad r \geq r_{c}
\end{gathered}
$$


In this article, $\sigma_{0}$ represents the diameter of $\mathrm{LJ}$ sphere and $\varepsilon$ means the strength of the interaction between beads. $r_{c}$ is the cutoff distance, which means that the beads separated by the distance larger than $r_{c}$ does not interact. The bonded interactions along each polymer chain are modeled via a simple harmonic potential $U_{\text {bond. }}$.

$$
U_{\text {bond }}(r)=\frac{1}{2} k\left(r-r_{0}\right)^{2}
$$

where $k$ is a parameter representing the bond strength, $r_{0}$ is a parameter representing the equilibrium bond distance.

The initial structures of chains were generated from the spatial distribution of segments obtained by phase separation simulation. The SCF calculation gave the spatial distribution of segments in the phase separated state. In the numerical calculation, the local volume fraction of segment $n: \phi_{n}(\mathbf{r})$ is given as $\phi_{n}(i, j, k)$, where $(i, j, k)$ is the index of the lattice point and $\phi_{n}(\mathbf{r})$ is calculated by the interpolation from the values of $\phi_{n}(i, j, k)$, of the nearby lattice points. For generating the initial chain configuration, the chains are grown using the density biased Monte Carlo procedure. The detail of the density biased Monte Carlo procedure can be found in Ref. (42). The initial MD models for tensile simulation were developed by replacing the spatial density distribution of PE segment obtained by the phase separation simulation to that of EPR in both morphology A and morphology B. The spatial density distributions of PP and talc were the same as those obtained in the phase separation simulation.

For structural relaxation by dynamics simulation, the excluded volume effect is introduced gradually by scaling the forces acting on atoms. The maximum force allowed to act on atoms is given and if the forces become larger than the maximum force, the force is scaled to that value. Then the maximum force for scaling is increased gradually, if the averaged force and the maximum force acting on each atom decrease in the relaxation process, and if the forces acting on all atoms become smaller than the initial maximum force for scaling, the relaxation is completed.

The simulation domain was a hexahedron $(56 L \times 32 L \times 14 L)$. The length of $\mathrm{z}$ direction was set as $14 L$ so that it should be larger than the inertial radius of the system. The polymer models are shown in Fig. 5. In this study, the polymer chain length of PP was set as 20 segments, leading to about 200 propylene monomers in one PP segment and about 340 ethylene monomers in one PE segment as shown in Table 4. In addition, talc was modeled one talc segment because talc did not have polymer chains. Here, EPR was modeled as triblock copolymer in order to make the interaction between PP and EPR strong. The number of polymer chains was decided such that the system density was 1.0. This resulted in 802 for polypropylene, 307 for EPR and 630 for talc, respectively. All the reduced units converted to SI units are shown in Table 5. Here, firstly, the unit length $L$, the unit density $\rho$ and the unit pressure $P$ were decided so that the simulation model should be similar to the actual PP/EPR/talc blend system, leading to the rest of the reduced units.

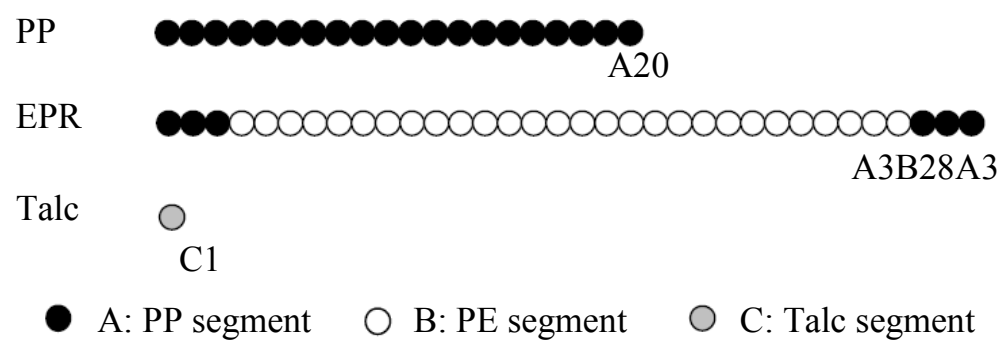

Figure $5 \mathrm{PP} / \mathrm{EPR} /$ talc polymer models 
Table 4 PP/EPR/talc polymer models

\begin{tabular}{cccc}
\hline & Number of monomers & Number of segments & Number of monomers in a segment \\
\hline \hline PP & 4529 & 20 & 227 \\
PP in EPR & 1219 & 6 & 204 \\
PE in EPR & 9567 & 28 & 342 \\
\hline
\end{tabular}

Table 5 Reduced units in SI unit

\begin{tabular}{cc}
\hline Reduced units & SI units \\
\hline \hline$L$ & $140 \mathrm{~nm}$ \\
$\rho$ & $0.945 \times 10^{3} \mathrm{~kg} / \mathrm{m}^{3}$ \\
$P$ & $12.658 \mathrm{MPa}$ \\
$M$ & $2.593 \times 10^{-24} \mathrm{~kg}$ \\
$\tau$ & $1.21 \mathrm{ps}$ \\
$u_{0}$ & $2.091 \times 10^{10} \mathrm{~J} / \mathrm{mol}$ \\
$T$ & $2.515 \times 10^{9} \mathrm{~K}$ \\
\hline
\end{tabular}

The bonding strengths of PP, PE and PP-PE segments were determined mainly based on the apparent elastic moduli of PP and EPR because the bonding strength is strongly related to the stiffness, in general. According to Ref. (38), the apparent elastic modulus of PP was about $1 \mathrm{GPa}$, and that of EPR ranged from 0.01 to $0.1 \mathrm{GPa}$. EPR consisted of both bonding connections, between PE and PE segments, and PP and PE segments, as illustrated in Fig. 7. Therefore, their bond strengths were determined as one tenth of that of PP segments. The strength of the L-J potential was identified based on the tensile strengths of PP and EPR. The tensile strength of PP ranges from 20 to $40 \mathrm{MPa}$ and that of EPR are between 10 and 20 $\mathrm{MPa}^{(34)}$. As a result, the strength of the L-J potential of PP segments was twice as large as that of PE segments. The strengths between PP and PE segments were determined by assuming that the interaction between the same kinds of segments should be larger than that between the different types of segments. It was also assumed that the strengths between talc and PP (or PE) segments should be smaller than that between PP and PE segments. The experimental observation ${ }^{(32,39)}$, that the debonding between in-organic fillers and $\mathrm{PP} /$ elastomers blends caused the plastic deformation and extensive cavitation, indicates that these assumption should be reasonable qualitatively. The identified parameters are shown in Tables 6 and 7.

Table 6 Bonding strengths

\begin{tabular}{|c|c|c|}
\hline \multicolumn{2}{|c|}{ Bonding pair } & Bond strength $k$ \\
\hline $\mathrm{A}$ & $\mathrm{A}$ & $300 u_{0} / L^{2}$ \\
\hline B & B & $30 u_{0} / L^{2}$ \\
\hline A & B & $30 u_{0} / L^{2}$ \\
\hline
\end{tabular}

Table 7 Strengths of interaction and cut-off length of LJ potential

\begin{tabular}{ccccc}
\hline \multicolumn{2}{c}{ Bonding pair } & $\varepsilon$ & $\sigma_{0}$ & $r_{\mathrm{c}}$ \\
\hline \hline $\mathrm{A}$ & $\mathrm{A}$ & $2 u_{0}$ & $1 L$ & $1.3 L$ \\
$\mathrm{~B}$ & $\mathrm{~B}$ & $1 u_{0}$ & $1 L$ & $2 L$ \\
$\mathrm{C}$ & $\mathrm{C}$ & $20 u_{0}$ & $0.891 L$ & $1.3 L$ \\
$\mathrm{~A}$ & $\mathrm{~B}$ & $0.75 u_{0}$ & $1 L$ & $2 L$ \\
$\mathrm{~A}$ & $\mathrm{C}$ & $0.1 u_{0}$ & $1 L$ & $2 L$ \\
$\mathrm{~B}$ & $\mathrm{C}$ & $0.1 u_{0}$ & $1 L$ & $2 L$ \\
\hline
\end{tabular}

The monomers in the simulation domain were evolved through time using the velocity Verlet integration algorithm. The numbers of calculating steps were 10,000 for relaxation with a time step of $0.012 \tau$ and $1,000,000$ for tensile deformation with a time step of $0.01 \tau$. Periodic boundaries were applied in the $\mathrm{x}, \mathrm{y}$ and $\mathrm{z}$ directions for relaxation and in the $\mathrm{y}$ and 
$\mathrm{z}$ directions for tensile deformation because the affine deformation in $\mathrm{x}$ direction was applied to the unit cell in the tensile deformation. The increment of strain was $10^{-6}$ in each calculating step. During the elongation, the stress was calculated based on Virial theorem.

Both relaxation and tensile simulations were conducted in the NVT ensemble using a Langevin thermostat ${ }^{(40,41)}$ with a friction of 0.5 and a set-point temperature of $0.1 T$. The temperature was controlled by the friction constant and the thermal noises. This was equivalent to the Langevin equation.

$$
m_{i} \frac{d^{2} \mathbf{r}_{i}}{d t^{2}}=F_{i}-\mu \frac{d \mathbf{r}_{i}}{d t}+\mathbf{W}_{i}(t)
$$

where $r_{i}$ is the coordinates of an atom $i, m_{i}$ is the mass of an atom $i, F_{i}$ is the force acting on an atom $i, \mu$ is the friction constant, $\boldsymbol{W}_{i}(t)$ is Gaussian white noise given by

$$
\left\langle\mathbf{W}_{i}(t) \mathbf{W}_{j}\left(t^{\prime}\right)\right\rangle=2 k_{B} T m_{i} \mu \delta_{i j} \mathbf{I} \delta\left(t-t^{\prime}\right),
$$

where $k_{B}$ is the Bolzman constant and $T$ is the current temperature.

The tensile strain rate in the $\mathrm{x}$-direction was $10^{-5}(1 / \tau)$. Because of the coarse-grained nature, the strain rate was much faster than the quasi-static deformation. Especially, the reduced length was much larger than the conventional coarse MD models, leading to very short time duration. However, it is validated that the total potential energy changed within small range $(0.05 \%$ of the total energy) during the tensile deformation, and the change of the potential energy was almost the same as that of the initial structural relaxation calculation. In addition, it is shown that the structure of the current model was relaxed fully during deformation in the snapshots of the morphological deformation. Moreover, it was confirmed that the maximum stresses of the present model were approximately similar to the simulated ones where the applied tensile deformation rates were 0.1 and 0.01 times as slow as the present model with the smallest interfacial strengths. Thus, it is considered that the current model can be similar to the quasi-static deformation process qualitatively. The simulation code used in this study was the standard coarse-grained molecular dynamics model with public domain meso-scale simulation code COGNAC $^{(42)}$ inside an integrated simulation system for soft materials OCTA ${ }^{(35)}$.

In this study, the qualitative analyses of the deformation mechanism and the damage evolution in two types of the PP/EPR/talc blend systems were mainly focused. In addition, the effects of the interfacial strengths of PP-EPR and PP-talc in the morphology A, and those of PP-EPR and EPR-talc in the morphology $\mathrm{B}$ on the tensile mechanical properties were investigated. For investigating the effect of PP-EPR interface in both morphologies A and $\mathrm{B}$, the strengths of the interaction between PP and EPR were changed as $0.075 u_{0}$, $0.1875 u_{0}, 0.375 u_{0}, 0.5625 u_{0}, 0.75 u_{0}, 0.9 u_{0}, 1.05 u_{0}, 1.2 u_{0}, 1.35 u_{0}$ and $1.5 u_{0}$ while the rest of the parameters were kept same as what were shown in Tables 6 and 7 where the indexes A, B and C mean PP, PE and talc segments, respectively. In the case of studying the effects of PP-talc interface in the morphology A and EPR-talc interface in the morphology B, the strengths of PP-talc interface and EPR-talc interface were parametrically changed as $0.01 u_{0}$, $0.025 u_{0}, 0.05 u_{0}, 0.075 u_{0}, 0.1 u_{0}, 0.12 u_{0}, 0.14 u_{0}, 0.16 u_{0}, 0.18 u_{0}$ and $0.2 u_{0}$ with the same parameters as shown in Tables 6 and 7.

\section{Results and Discussions}

\subsection{Tensile Deformation Behaviors up to Failure in both Morphologies}

Figure 6 shows the initial phase morphologies of two-type morphologies after relaxation where the blue-color area is PP, the green-color area is EPR and the red-color area is talc particles, respectively. In the morphology A, it is clearly shown that the EPR particles and the talc are separately dispersed as islands in the PP matrix. On the contrary, 
the morphology B showed that the talc and the EPR particle constructed the core-shell structure in the PP matrix.

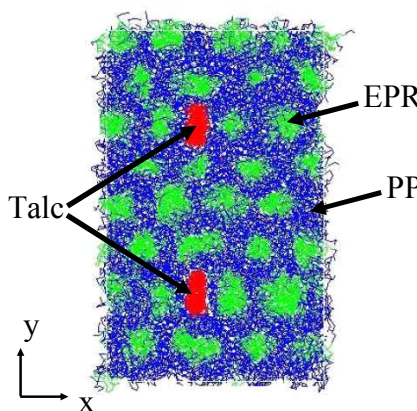

(a) Morphology A

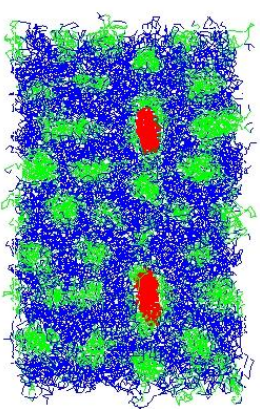

(b) Morphology B

Figure 6 Initial phase morphologies before tensile deformation

Figure 7 shows the snap shots of deformation process of the morphologies A and B. At the beginning of tensile deformation, the elongations in the PP matrix and the EPR particles were uniform. Then, at the tensile nominal strain of 0.04 , the debonding occurred at the interface between PP and talc phases in the morphology A, and the micro damage was nucleated at the interface between the PP and the talc phases. On the contrary, the micro damage started growing at the interface between the EPR particles and the talc phase in the morphology B. Next, the voids around the talc particles induce the cavitations of the adjacent EPR particles in the morphology A while the cavitations occurred mainly in the EPR particles encapsulating talc in the morphology B, respectively. Finally, the molecular chains of PP and EPR phases were fibrillated in both morphologies. In our previous study ${ }^{(32)}$, it was shown that the simulated deformation and damage evolution mechanism of the morphology A was similar to the in situ TEM microstructural observation of the thin specimen under tensile loading. This indicated that the micro-damage evolution process was successfully captured by the present MD model, leading to the clear understanding of the micro-damage evolution in both morphologies A and B of PP/EPR/talc blend systems.

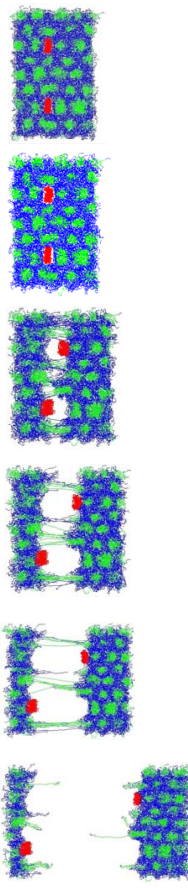

(a) Morphology A

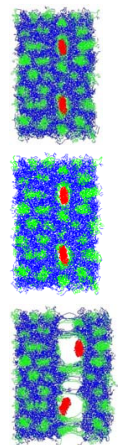

Strain 0.2

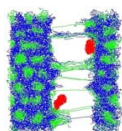

Strain 0.4

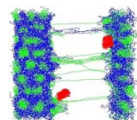

Strain 0.6

Strain 1.0

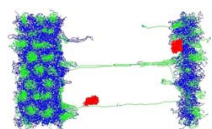

(b) Morphology B

Figure 7 Snapshots of microstructural deformation processes in morphologies A and B 


\subsection{Tensile Stress-Strain Relationships in both Morphologies}

Figure 8 shows the tensile stress-strain curves of the morphologies A and B. The clear differences between them were the maximum stress and the stress softening after the peak stress. The maximum stress of morphology B was a little larger than that of morphology A. As shown in Fig. 7, it is considered that the different initial damage location would lead to the different damage evolution process. After the peak stress, the damage evolved by the cavitation of the EPR particles in the morphology A. On the contrary, the EPR particles were not cavitated fully because of the encapsulated structure, leading to the strong stress softening after the peak stress in the morphology B. The comparison of the mechanical properties is shown in Fig. 9. The apparent elastic modulus was a little larger in the separated morphology (morphology A) than the core-shell structure (morphology B), which was the same result as Refs. (28-32). On the other hand, the maximum stress of the core-shell morphology (morphology B) was larger than that of the separated morphology (morphology A), which agreed with the results obtained by Kolarik et al. ${ }^{(31)}$ and Premphet et al. ${ }^{(32)}$. The strain energy up to the strain of 1.0 was about $20 \%$ larger in the separated morphology (morphology A) than the core-shell morphology (morphology B). This trend also coincided with Refs. $(31,32)$.

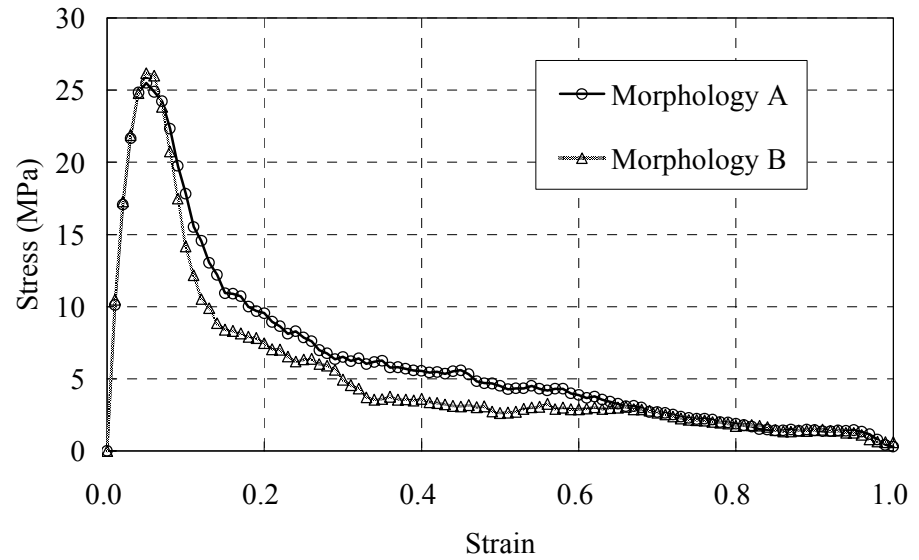

Figure 8 Tensile stress-strain relationships in morphologies A and B

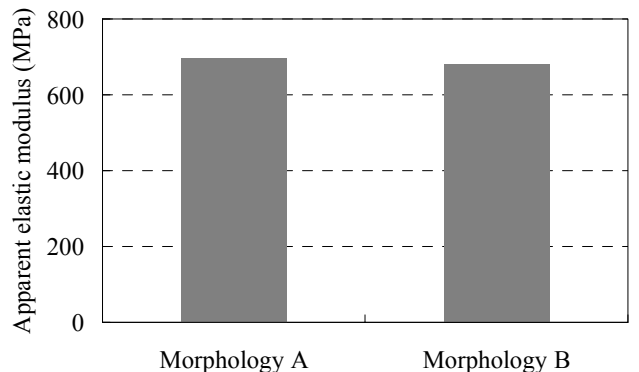

(a)

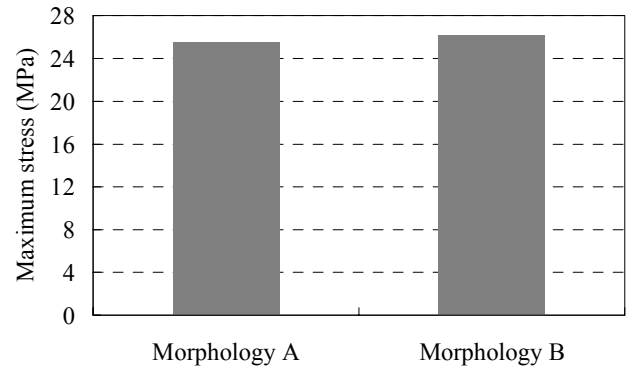

(b)

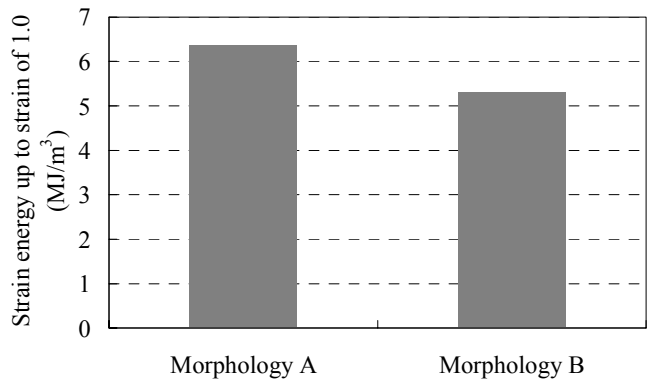

(c)

Figure 9 Summarized mechanical properties in morphologies A and B 


\subsection{Effects of Interfacial Strengths on Stress-Strain Curves in both Morphologies}

The effects of the interfacial strengths of PP-EPR and PP-talc in the morphology A, and those of PP-EPR and EPR-talc in the morphology B were investigated by changing the strength parameter of interaction, $\varepsilon$. Figures 10(a) and 11(a) show the stress-strain curves of various interfacial strengths of the PP phase and the EPR particles in the morphologies A and B, respectively, while Figures 10 (b) and 11 (b) show the stress-strain relationships with various interfacial strengths of the PP-talc in the morphology A and those of the EPR-talc in the morphology B. In both morphologies A and B, as the interfacial strength of PP-EPR phases increased, the stiffness and maximum stress increased with the decreasing yield strain. Here, the yield strain was defined as the strain at the maximum stress. At the yield strain, the debonding occurred along the interface between the PP and talc phases. It is considered that the deformation was localized at the interfaces of PP-talc in the morphology $A$ and EPR-talc in the morphology B with the increased PP-EPR interfacial strength, leading to the decrease of the yield strain in both morphologies. On the contrary to Figures 10(a) and 11(a), the trend of the stress-strain curves in Figs. 10 (b) and 11 (b) did not change drastically in each case for both morphologies even though the talc interfaces were strengthened. It is clear that the tensile stress-strain relationship should depend much more strongly on the interfacial strengths of PP-EPR than those of PP-talc interface and EPR-talc interface in the present two types of morphologies.

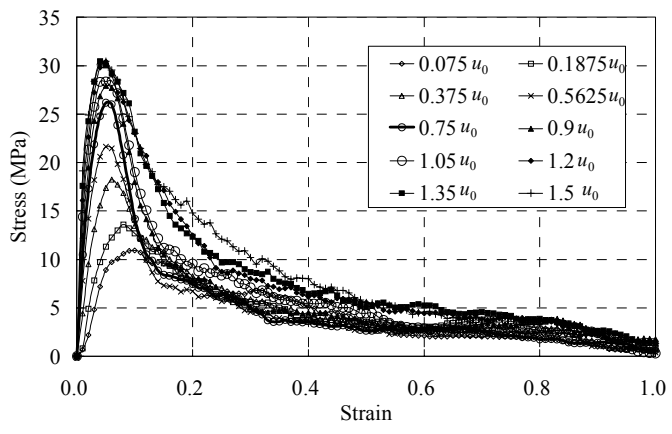

(a-i) Whole range of strain

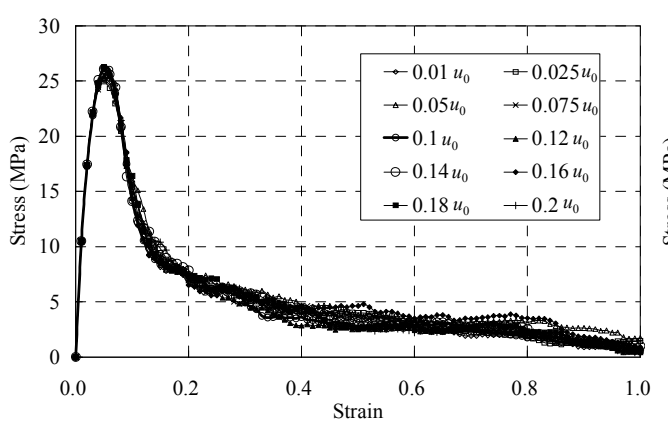

(b-i) Whole range of strain

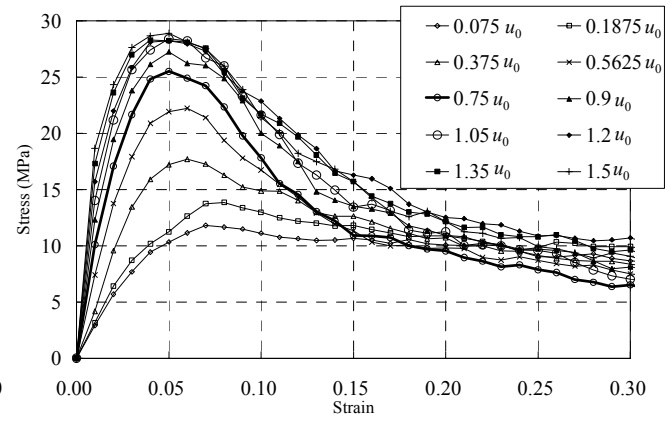

(a-ii) Strain up to 0.30

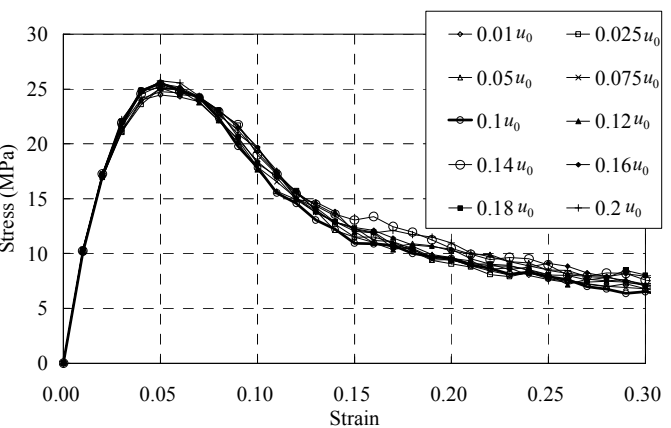

(b-ii) Strain up to 0.30

Figure 10 Stress-strain curves with various interfacial strengths in morphology A

(a-i) and (a-ii): Interfacial strengths of PP-EPR

(b-i) and (b-ii): Interfacial strengths of PP-talc 


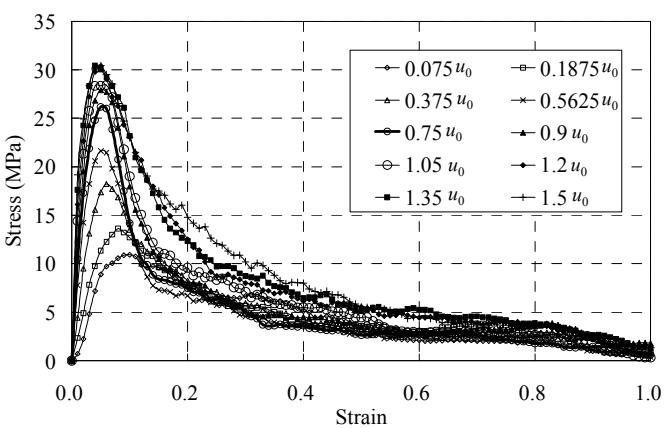

(a-i) Whole range of strain

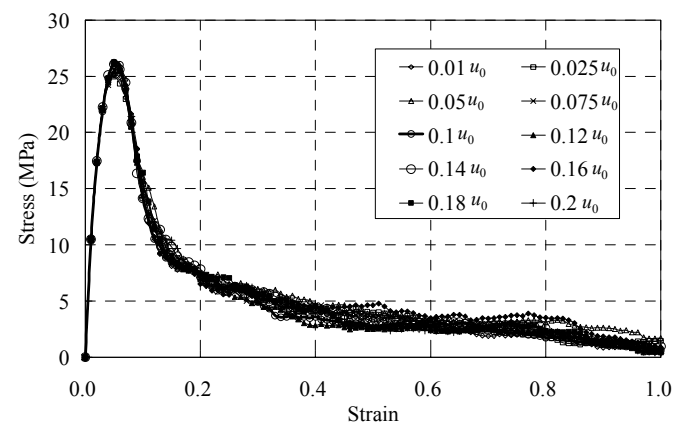

(b-i) Whole range of strain

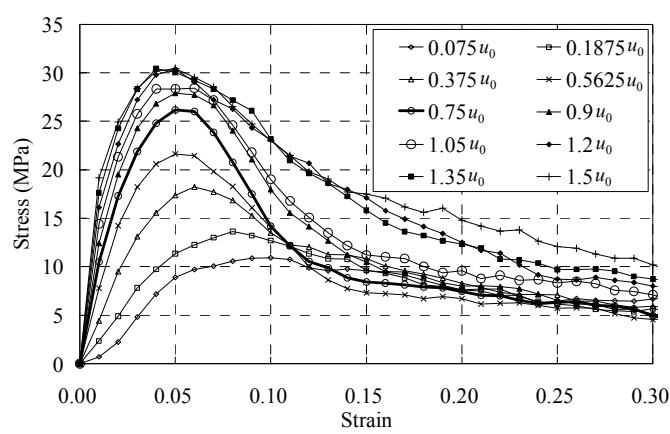

(a-ii) Strain up to 0.30

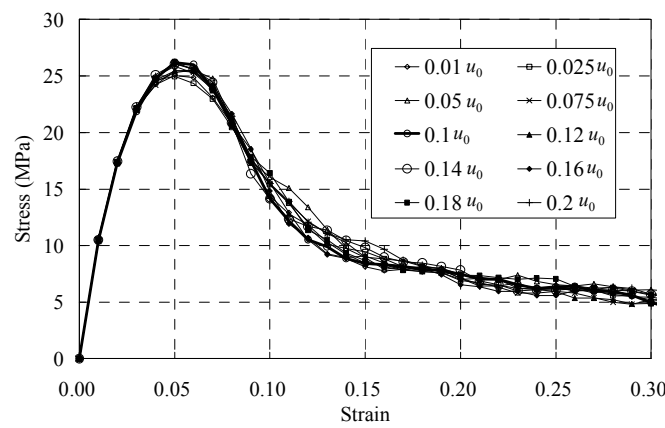

(b-ii) Strain up to 0.30

Figure 11 Stress-strain curves with various interfacial strengths in morphology B

(a-i) and (a-ii): Interfacial strengths of PP-EPR

(b-i) and (b-ii): Interfacial strengths of EPR-talc

\subsection{Effects of Interfacial Strengths on Mechanical Properties in both Morphologies}

Figure 12 shows the apparent elastic modulus plotted against the various normalized interfacial strengths of PP-EPR and PP-talc in the morphology A, and those of PP-EPR and EPR-talc in the morphology B. The apparent elastic modulus was defined as the slope of the tensile stress-strain curve at the strains between 0.01 and 0.02 in this study. Here, the interfacial strengths of PP-EPR, PP-talc and EPR-talc were normalized by $0.75 u_{0}, 0.1 u_{0}$ and $0.1 u_{0}$, respectively. It was found that the interfaces of PP-talc and EPR-talc did not have strong effect on the apparent elastic modulus in both morphologies. In the present $\mathrm{PP} / \mathrm{EPR} / \mathrm{talc}$ blends where the volume fractions of EPR and talc were about $38 \%$ and $3 \%$ respectively, it is considered that the interfacial area of PP-EPR was much larger than those of PP-talc and EPR-talc, leading to the strong effect of PP-EPR interfacial strength on the macroscopic apparent elastic modulus.

It appeared that the apparent elastic modulus of morphology A was a little larger than that of the morphology B at the normalized interfacial strengths of PP-EPR below 1.4. Further increase of the interfacial strength of PP-EPR, e.g. 1.6, 1.8 and 2.0, made the apparent elastic modulus of the morphology B a little larger than that of morphology A. As reported in the previous paper ${ }^{(33)}$, the strong interface between the PP phase and the EPR particles localized the deformation at the interface of PP-talc, leading to the decrease of the apparent elastic modulus. On the contrary, in the morphology B, it is considered that the strong PP-EPR interface transferred the external stress directly to the talc particles, leading to the larger elastic modulus than the morphology A. The difference of the microstructural deformation would make the different trend of the apparent elastic modulus at the interfacial strengths of PP-EPR above 1.4. The literatures reported that the elastic modulus of the separated morphology was larger than that of the core-shell structure ${ }^{(28)-(32)}$, which was similar to the present results at the interfacial strength of PP-EPR smaller than 1.4. It was found that the effect of the morphological difference on the apparent elastic modulus 
got large as the interfacial strength of PP-EPR becomes substantial. Note, that the obtained trend of the apparent elastic modulus would not change even though both the interfacial strengths of PP-EPR and talc-PP (or EPR) were changed parametrically at the same time because the effects of the talc interfaces were much smaller than those of PP-EPR interfaces.

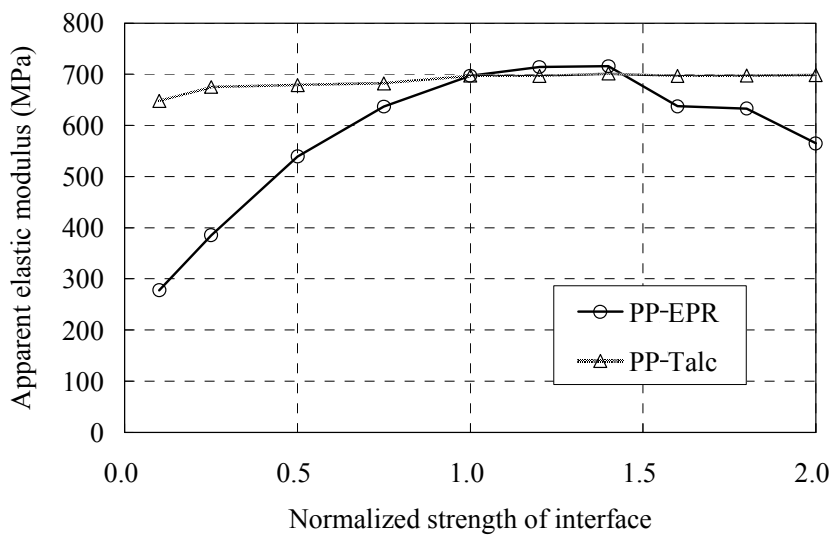

(a) Morphology A

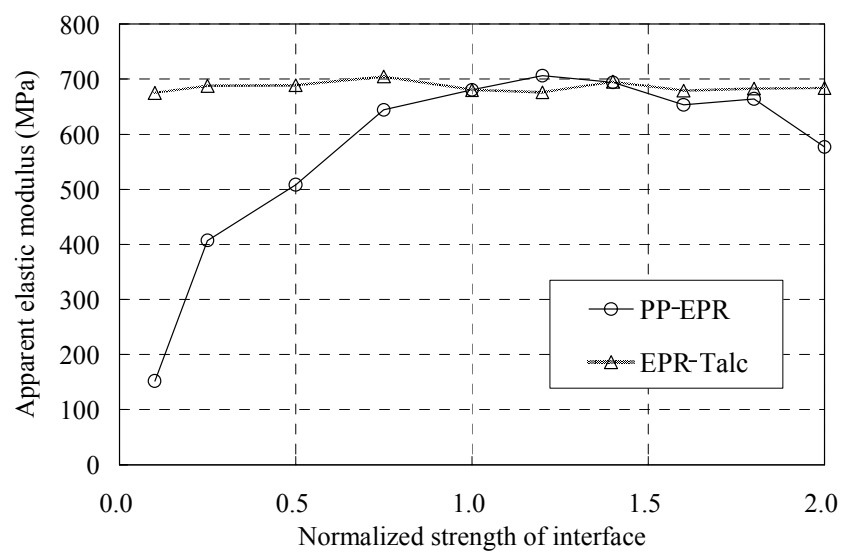

(b) Morphology B

Figure 12 Effects of interfacial strengths on apparent elastic modulus

Figure 13 shows the maximum stress plotted against the various normalized interfacial strengths of PP-EPR and PP-talc in the morphology A, and those of PP-EPR and EPR-talc in the morphology B. Here, the interfacial strengths of PP-EPR, PP-talc and EPR-talc were normalized by $0.75 u_{0}, 0.1 u_{0}$ and $0.1 u_{0}$, respectively. Figure 14 shows the snapshots of the microstructural deformation when the normalized interfacial strengths of PP-EPR were 0.1 and 2.0 in the morphologies $\mathrm{A}$ and $\mathrm{B}$ at the tensile strain where the stress reached to the maximum value. In both morphologies, the maximum stresses were obtained when the debondings occurred at the PP-talc interface in the morphology A, and the EPR-talc interface in the morphology B.

In the same as the apparent elastic modulus, the PP-EPR interface had stronger contribution on the maximum stress than the PP-talc and EPR-talc interfaces. It appeared that the maximum stress of the morphology B got substantially larger than that of the morphology A as the normalized interfacial strength of PP-EPR increased in the range more than 1.5. It is considered that the strong PP-EPR interface transferred the external stress more directly to the talc particles in the morphology $\mathrm{B}$, leading to the larger maximum stress than the morphology A at the interfacial strength larger than 1.5. 


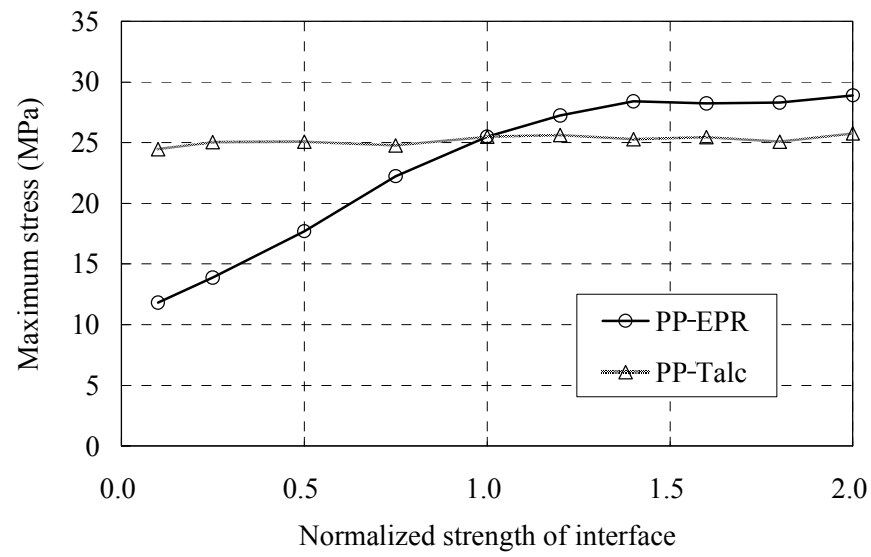

(a) Morphology A

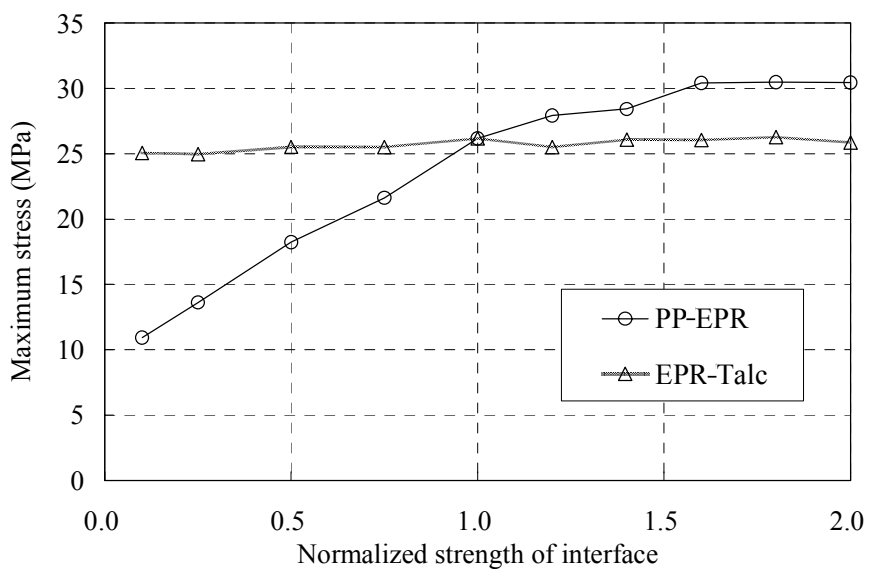

(b) Morphology B

Figure 13 Effects of interfacial strengths on maximum stress

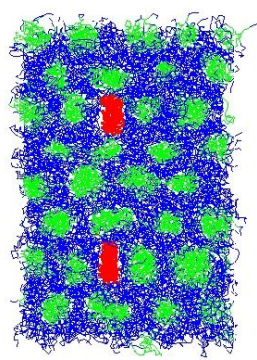

(i) 0.1

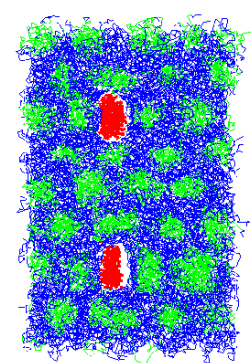

(ii) 2.0

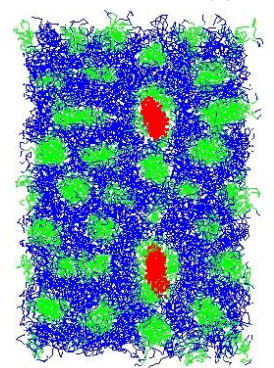

(i) 0.1

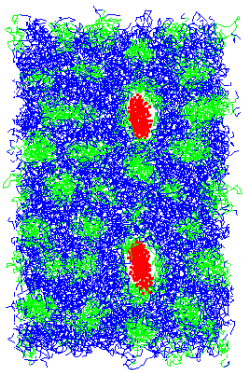

(ii) 2.0

(b) Morphology B

Figure 14 Snapshots of micro deformation in PP-EPR interfacial strengths of 0.1 and 2.0 at maximum stress 
Figure 15 shows the strain energy up to the tensile strain of 1.0 plotted against the various normalized interfacial strengths of PP-EPR, PP-talc and EPR-talc. Again, the interfacial strengths of PP-EPR, PP-talc and EPR-talc were normalized by $0.75 u_{0}, 0.1 u_{0}$ and $0.1 u_{0}$, respectively. As the same as Figs. 12 and 13, the interfacial strengths of PP-talc and EPR-talc showed the weak contributions on the absorbed strain energy in both types of morphologies. On the contrary, the PP-EPR interface had more strong influence on the absorbed strain energy in the morphology B than that in the morphology A, which was different trends from the apparent elastic modulus and the maximum stress. This trend indicates that it is important to arrange the interfacial strength of PP-EPR in the material designing, especially in the case of the core-shell type of morphology. In the base models where the normalized interfacial strengths were 1.0, the morphology A showed the larger absorbed strain energy than the morphology B. However, when the normalized interfacial strength of PP-EPR was above 1.6, then the absorbed strain energy of the morphology B could be improved to the similar level to the morphology A. These numerical results indicate that the absorbed strain energy would be more sensitive to both the interfacial strength of PP-EPR and the morphological types such as the separated and the core-shell structures. It is considered that this trend could be the reason why the contrasting experimental results were obtained in both morphological types ${ }^{(28-32)}$.

Figure 16 shows the snapshots of the cases where the normalized interfacial strengths of PP-EPR were 0.1 and 2.0 in the morphologies A and B at the tensile strain of 0.2 . At this tensile strain, the large difference of the stress softening was investigated in the stress strain curves as shown in Figs. 10 and 11. As shown in Fig. 16, there were two types of deformation modes in both morphologies. When the normalized interfacial strength of PP-EPR was 0.1, the debonding occurred at the interface of PP-EPR as well as the talc interfaces, leading to the drastic decrease of the macroscopic stress without depending on the morphological types. On the contrary, the debonding occurred around talc particles in both morphologies when the normalized interfacial strength of PP-EPR was 2.0, leading to the ductile mode.

With the increasing interfacial strength of PP-EPR, both PP and EPR phases were fibrillated in the same bundles, as shown in Fig. 16 (b-ii), leading to the increase of the strain energy of the morphology B. On the other hand, the fibrils were not well developed with the small interfacial strength of PP-EPR as shown in Fig. 16 (b-i), leading to the small strain energy in the morphology B. In the morphology A, the deformable PP phases were larger than the morphology B because the direct interface between PP phase and talc particles transferred the applied stress to the PP phase around talc particles even though the PP-EPR interface was weak. This is how the morphology A kept the strain energy larger than $6 \mathrm{MJ} / \mathrm{m}^{3}$ in the whole range of the interfacial strength of PP-EPR.

In the realistic case, it is very hard to increase the interfacial strengths of PP-talc and EPR-talc compared to the PP-EPR interface. Thus, it is considered that the increasing the interfacial strength of PP phase and EPR particles is very important task for improving the mechanical properties in the PP/EPR/talc blend system in both types of morphologies. 


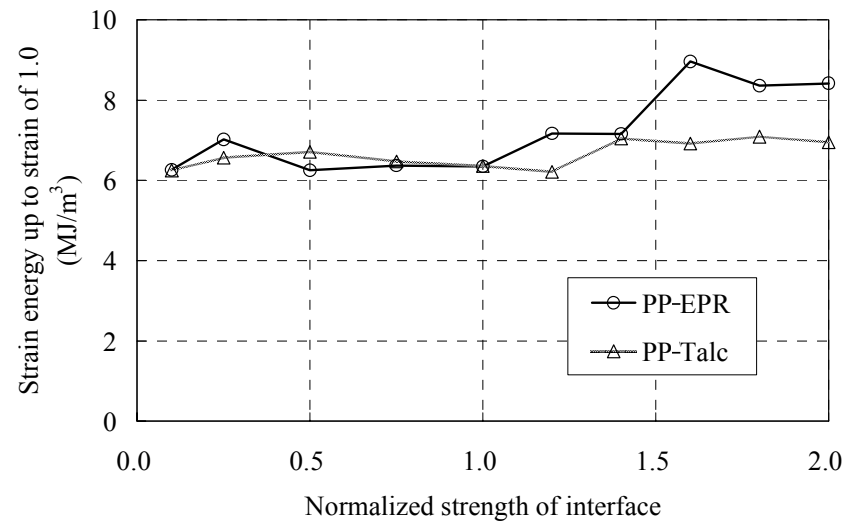

(a) Morphology A

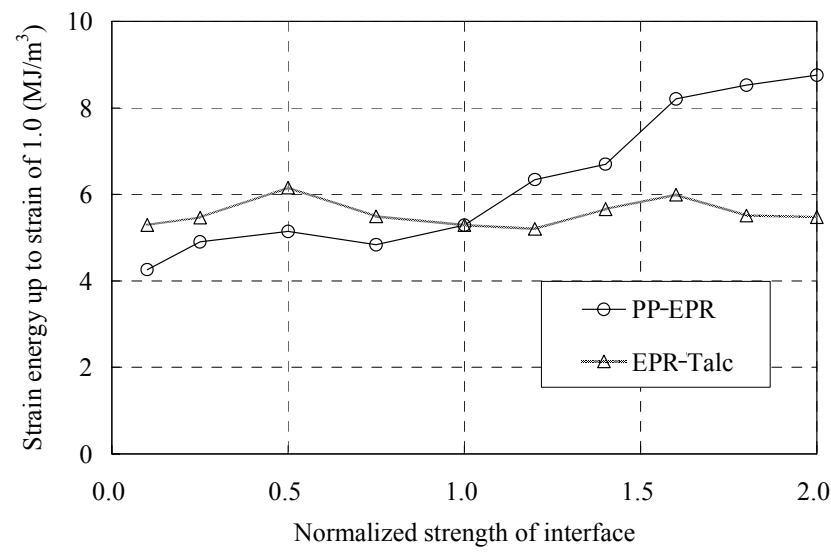

(b) Morphology B

Figure 15 Effects of interfacial strengths on strain energy

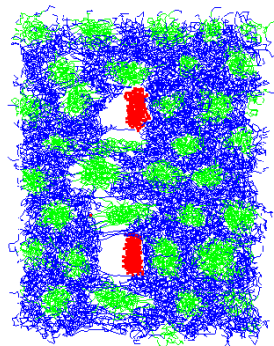

(i) 0.1

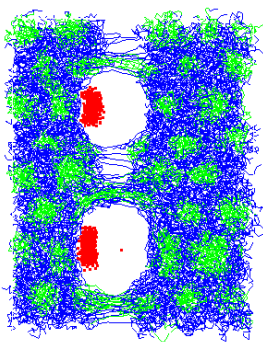

(ii) 2.0

(a) Morphology A

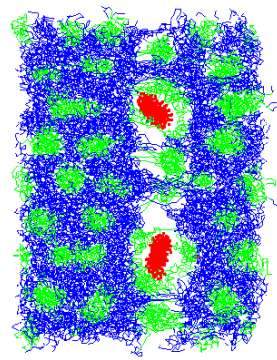

(i) 0.1

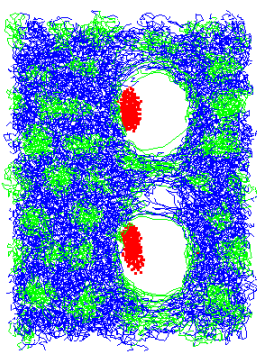

(ii) 2.0

(b) Morphology B

Figure 16 Snapshots of micro deformation in PP-EPR interfacial strengths of 0.1 and 2.0 at nominal strain of 0.2

\section{Conclusions}

In order to clarify the influences of the morphology and the interfacial strength on the 
microstructural deformation and micro-damage evolution process, a coarse-grained molecular dynamics simulation was conducted on a mesoscopic specimen of PP blended with EPR and talc under uniaxial tension. The studied morphologies were two types. The first morphology was composed of three independent phases of PP, EPR and talc. The second morphology consisted of core-shell structure of EPR and talc in PP matrix. In addition, the effects of the interfacial strength of PP-EPR and PP-talc in the first morphology, and those of PP-EPR and EPR-talc in the second morphology on the microstructural deformation, damage evolution and macroscopic mechanical properties were investigated. The followings are the conclusions of this study:

1. The micro damage initiated at the interface of talc without depending on the morphological types such as the separated and the core-shell structures.

2. The apparent elastic modulus was a little larger in the separated morphology than that in the core-shell type.

3. The maximum stress depended more strongly on the interfacial strength of PP-EPR than the morphological types.

4. The absorbed strain energy was sensitive to the interfacial strength of PP-EPR and the morphological types such as the separated and the core-shell structures.

5. To improve the stiffness, the maximum stress and ductility, it is more effective to increase the interfacial strength between PP-EPR, compared to the interfacial strengths of PP-talc and EPR-talc.

6. If the interfacial strengths of PP-EPR is very strong, the stiffness, the maximum stress and the strain energy can be larger in the core-shell morphology than the separated morphology.

\section{References}

(1) Mae H., Takada K., Takahashi J., Yamamoto T., Honda R\&D Technical Review, Vo.16, No.1 (2004), pp.165-172.

(2) Mae H., K. Kishimoto, Transactions of Society of Automotive Engineers of Japan, Vol.38, No.3 (2007), pp.63-68.

(3) Petrovicova R., Knight R., Schadler LS., Twadowski TE., Journal of Applied Polymer Science, Vo.78, No.13 (2000), pp.2272-2289.

(4) Petrovics Z.S., Javni I., Waddon A., Banhegi G., Journal of Applied Polymer Science, Vol.76, No.2 (2000), pp.133-151.

(5) Mae H., Omiya M., Kishimoto K., Journal of Solid Mechanics and Materials Engineering, Vol.2, No.2 (2008), pp.254-268.

(6) Gonzalez J., Albano C., Ichazo M., Diaz B., European Polymer Journal, Vol.38, No.12 (2002), pp.2465-2475.

(7) Mae H., Omiya M., Kishimoto K., Journal of Applied Polymer Science, Vol.110 (2008), pp.1145-1157.

(8) Ferrage E., Martin F., Boudet A., Petit S., Fourty G., Jouffret F., Micoud P., De Parseval P., Salvi S., Bourgerette C., Ferret J., Saint-Gerard Y., Buratto S., Fortune J. P., Journal of Materials Science, Vol.37, No.8 (2002) pp.1561-1573.

(9) Zihlif A.M., Ragosta G., Materials Letters, Vol.11, No.10-12 (1991), pp.368-372.

(10) Zhou Y., Mallick P.K., Polymer Engineering and Science, Vol.42, No.12 (2002), pp.2449-2460.

(11) Zhou Y., Rangari V., Mahfuz H., Jeelani S., Mallick P.K., Materials Science and Engineering A, Vol.402 (2005), pp.109-117.

(12) Hambir S., Bulakh N., Jog J.P., Polymer Engineering and Science, Vol.42, No.9 (2002), pp.1800-1807.

(13) Hadal R.S., Dasari A., Rohrmann J., Misra R.D.K., Materials Science and Engineering A, 
Vol.372 (2004), pp.296-315.

(14) Hadal R.S., Misra R.D.K., Materials Science and Engineering A, Vol.374 (2004), pp.374-389.

(15) Liang J.Z., Li R.K.Y., Journal of Applied Polymer Science, Vol.77, No.2 (2000), pp.409-417.

(16) Wei G.-X., Sue H.-J., Chu J., Huang C., Gong K., Journal of Materials Science, Vol.35, No.3 (2000), pp.555-566.

(17) Velasco J.I., De Saja J.A., Martinez A.B., Journal of Applied Polymer Science, Vol.61 (1996), pp.125-132.

(18) Suh C.H., White J.L., Polymer Engineering and Science, Vol.36, No.17 (1996), pp.2188-2197.

(19) Mae H., Omiya M., Kishimoto K., Transactions of the Japan Society for Computational Methods in Engineering, Vol.7, No.2 (2008), pp.207-212.

(20) Stamhuis J.E., Polymer Composites, Vol.5, No.3 (1984) pp.202-207.

(21) Mae H., Omiya M., Kishimoto K., Journal of Applied Polymer Science, Vol.107 (2008), pp.3520-3528.

(22) Mae H., Omiya M., Kishimoto K., Journal of the Society of Materials Science Japan, Vol.57, No.9 (2008), pp.951-958.

(23) Mae H., Omiya M., Kishimoto K., Journal of Applied Polymer Science, Vol. 110 (2008), pp.3941-3953.

(24) Garcia-Martinez J.M., Laguna O., Areso S., Collar E.P., European Polymer Journal, Vol.38 (2002) pp.1583-1589.

(25) Garcia-Martinez J.M., Laguna O., Areso S., Collar E.P., Journal of Polymer Science: Part B: Polymer Physics, Vol.40 (2002), pp.1371-1382.

(26) Velasco J.I., De Saja J.A., Martinez A.B., Journal of Applied Polymer Science, Vol.61 (1996), pp.125-132.

(27) Wah C.A., Choong L.Y., Neon G.S., European Polymer Journal, Vol.36 (2000), pp.789-801.

(28) Hornsby R.P., Premphet K., Journal of Applied Polymer Science, Vol.70 (1998), pp.587-597.

(29) Kolarik J., Lednicky F., Jancar J., Pukanszky B., Polymer Communications, Vol.31 (1999), pp.201-204.

(30) Long Y., Shanks R.A., Journal of Applied Polymer Science, Vol.61 (1996), pp.1877-1885.

(31) Kolarik J., Jancar J., Polymer, Vol. 33 (1992), pp. 4961-4967.

(32) Premphet K., Horanont P., Journal of Applied Polymer Science, Vol.76 (2000), pp.1929-1939.

(33) Mae H., Omiya M., Kishimoto K., Journal of Solid Mechanics and Materials Engineering, Vol.2, No.8 (2008), pp.1018-1036.

(34) SUSHI User Manual, Version 6.0 (2005).

(35) Doi M., Macromolecular Symposia, Vol.195, No.1 (2003), pp.101-108.

(36) Evers O.A., Scheutjens J.M.H.M., Fleer G.J., Macromolecules, Vo.23, No.25 (1990), pp.5221-5233.

(37) Matsen M.W., Bates F.S., Macromolecules, Vol.29, No.4 (1996), pp.1091-1104.

(38) Ashby M., Shercliff H., Cebon D., Materials Engineering, Science, Processing and Design, (2007), pp.156, Elsevier.

(39) Michler G.H., Balta-Calleja F.J., Mechanical Properties of Polymers Based on Nanostructure and Morphology (2005), pp.393, Taylor \& Francs.

(40) Grest G.S., Kremer K., Physical Review A, Vol.33 (1986), pp.3628-3631.

(41) Frenkel, D., Smit, B., Understanding Molecular Simulation, $2^{\text {nd }}$ ed., (2001), Academic Press.

(42) COGNAC User Manual, Version 4.2.4 (2005). 